\title{
Microarray analysis of differentially expressed genes in L929 mouse fibroblast cells exposed to leptin and hypoxia
}

\author{
PING OUYANG $^{1 *}$, SEN WANG $^{2 *}$, HE ZHANG $^{3}$, ZHIGANG HUANG $^{3}$, \\ $\mathrm{PEI} \mathrm{WEI}^{1}$, YE ZHANG ${ }^{2}, \mathrm{ZHUGUO}^{4} \mathrm{WU}^{4}$ and TAO $\mathrm{LI}^{1}$ \\ ${ }^{1}$ Guangdong Provincial Key Laboratory of Medical Molecular Diagnostics, Dongguan Scientific Research Center; \\ ${ }^{2}$ Cancer Institute of Guangdong Medical University; ${ }^{3}$ Department of Epidemiology, School of Public Health; \\ ${ }^{4}$ The Second Clinical College, Guangdong Medical University, Dongguan, Guangdong 523808, P.R. China
}

Received February 4, 2016; Accepted January 26, 2017

DOI: $10.3892 / \mathrm{mmr} .2017 .6596$

\begin{abstract}
Leptin and hypoxia are pro-fibrotic factors involved in fibrogenesis, however, the gene expression profiles remain to be fully elucidated. The aim of the present study was to investigate the regulatory roles of leptin and hypoxia on the L929 mouse fibroblast cell line. The cells were assigned to a normoxia, normoxia with leptin, hypoxia, and hypoxia with leptin group. The cDNA expression was detected using an Agilent mRNA array platform. The differentially expressed genes (DEGs) in response to leptin and hypoxia were identified using reverse transcription-quantitative polymerase chain reaction analysis, followed by clustering analysis, Gene Ontology analysis and pathway analysis. As a result, 54, 1,507 and 1,502 DEGs were found in response to leptin, hypoxia and the two combined, respectively, among which 52 (96.30\%), 467 (30.99\%) and 495 (32.96\%) of the DEGs were downregulated. The most significant functional terms in response to leptin were meiosis I for biological process $(\mathrm{P}=0.0041)$ and synaptonemal complex for cell component $(\mathrm{P}=0.0013)$. Only one significant pathway responded to leptin, which was axon guidance $(\mathrm{P}=0.029)$. Flow cytometry confirmed that leptin promoted L929 cell proliferation. The most significant functional terms in response to hypoxia were ion binding for molecular function $(\mathrm{P}=7.8621 \mathrm{E}-05)$, glucose metabolic process for biological process $(\mathrm{P}=0.0008)$ and cell projection part for cell component $(\mathrm{P}=0.003)$. There were 12 pathways, which significantly responded to hypoxia $(\mathrm{P}<0.05)$ and the pathway with the highest significance was the chemokine signaling
\end{abstract}

Correspondence to: $\mathrm{Dr}$ Tao Li, Guangdong Provincial Key Laboratory of Medical Molecular Diagnostics, Dongguan Scientific Research Center, Guangdong Medical University, 1 Xincheng Road, Songshan Lake Science and Technology Industry Park, Dongguan, Guangdong 523808, P.R. China

E-mail: taoliby@hotmail.com

*Contributed equally

Key words: leptin, hypoxia, fibroblast, cell cycle, chemokine pathway ( $\mathrm{P}=0.0001)$, which comprised 28 genes, including $\mathrm{C}-\mathrm{C}$ motif ligand (CCL)1, C-X-C motif ligand (CXCL)9, CXCL10, son of sevenless homolog 1, AKT serine/threonine kinase 2, Rho-associated protein kinase 1, vav guanine nucleotide exchange factor 1, CCL17, arrestin $\beta 1$ and C-C motif chemokine receptor 2 . In conclusion, the present study showed that leptin and hypoxia altered the profiles of gene expression in L929 cells. These findings not only extend the cell spectrum of leptin on cell proliferation, but also improve current understanding of hypoxia in fibroblast cells.

\section{Introduction}

Tissue fibrosis alters the tissue architecture and leads to organ dysfunction, which is major contributor to morbidity and mortality rates worldwide (1). The progression of fibrosis is similar in different organs, which is characterized by the activation and abnormal proliferation of fibroblasts/myofibroblasts and extracellular matrix remodeling (2). However, the mechanism underlying fibrogenesis is complex. Infection with pathogenic organisms, epigenetic alterations, B cells, transforming growth factor (TGF) $\beta$ signaling and TGF $\beta /$ small mothers against decapentaplegic (SMAD) 33-independent mechanisms have been reported to be involved in the activation of myofibroblasts (3-7). Several exogenous factors are also involved in fibrogenesis, including leptin and hypoxia (8-10). Previous studies have shown that leptin stimulates the production of tissue inhibitor of metalloproteinase 1 via the Janus kinase (JAK)/signal transducer and activator of transcription (STAT) pathway to directly promote fibrogenesis in hepatic stellate cells (11). Liver fibrosis is decreased in leptin- or leptin receptor-deficient mice (12). The in vitro administration of leptin to primary cardiofibroblasts has been found to result in the significant stimulation of pro-collagen I $\alpha$ and also leads to a decrease in the gene expression of pro-matrix metalloproteinase- $8,-9$ and -13 at $24 \mathrm{~h}$, which results in heart fibrosis (13). In addition, leptin is involved in renal fibrosis (14). Hypoxia is also an established profibrotic factor $(9,15,16)$. In hepatic fibrosis, hypoxia acts as a major inducer of angiogenesis together with inflammation, and hepatic angiogenesis and fibrosis have been found to be closely associated in clinical and experimental conditions (8). 
Hypoxia was found to induce cardiac fibrosis by upregulating focal adhesion kinase in cardiac fibroblasts or in a mouse model of post-myocardial infarction (17). Hypoxia-induced deoxycytidine kinase contributes to epithelial proliferation in pulmonary fibrosis (18). Hypoxia is also involved in hepatic fibrosis through potentiating the activity of hypoxia inducible factor-1 $\alpha$, either directly or through the epidermal growth factor (EGF)/mitogen-activated protein kinase (MAPK) and vascular endothelial growth factor (VEGF)/AKT pathway (8). However, the effects of leptin and hypoxia on fibrosis remain to be fully elucidated. The aim of the present study was to investigate the gene expression profiles of leptin and hypoxia in mouse fibroblast cell line L929 and analyze their possible biological functions in fibrosis processes. The present study showed that leptin and hypoxia altered the profiles of gene expression in L929 cells. The pro-fibrotic roles of leptin may be through promoting L929 cell proliferation; whereas hypoxia affected L929 cell function primarily through the chemokine signaling pathway.

\section{Materials and methods}

Cell culture and treatment. The L929 mouse fibroblast cells, purchased from the Kunming Cell Bank (Kunming, China) were cultured in Dulbecco's modified Eagle's medium with $5 \%$ fetal bovine serum (Gibco; Thermo Fisher Scientific, Inc., Waltham, MA, USA) in a humidified $5 \% \mathrm{CO}_{2}$ incubator at $37^{\circ} \mathrm{C}$. The $\mathrm{L} 929$ cells were used for all the following experiments. For leptin treatment, mouse recombinant leptin (200 ng/ml; Sigma-Aldrich; Merck Millipore, Darmstadt, Germany) was added to the cells. For hypoxic treatment, the L929 cells were transferred to a hypoxia chamber (MIC101; Billups-Rothenberg, Inc., Del Mar, CA, USA) where the total oxygen concentration was reduced to $<1 \%$.

cDNA expression array. The cells were cultured in $10 \mathrm{~cm}$ plates with $2.5 \times 10^{6}$ cells and divided into the following four groups: Group I, cells cultured in normoxia; Group II, cells treated with leptin in normoxia; Group III, cells cultured in hypoxia; Groups IV, cells treated with leptin in hypoxia. Every group included three parallel samples and the treatment temperature was $37^{\circ} \mathrm{C}$. After $24 \mathrm{~h}$, the cells were collected and placed in TRIzol reagent (Invitrogen; Thermo Fisher Scientific, Inc.), respectively.

Total RNA was extracted using TRIzol reagent according to the manufacturer's protocol. The RNA was purified using the mirVana miRNA isolation kit (Ambion; Thermo Fisher Scientific, Inc.). The RNA quality from each sample was assessed by visualization of the $28 \mathrm{~S} / 18 \mathrm{~S}$ ribosomal RNA ratio using $1 \%$ formaldehyde denaturing gel electrophoresis. The Agilent mouse mRNA array was designed with eight identical arrays per slide (8x60K format), with each array containing probes interrogating 39,430 Entrez Gene RNAs. The array also contained 1,280 Agilent control probes. The arrays were hybridized in an Agilent hybridization oven overnight at a rotation speed of $40 \mathrm{~g}$ at $42^{\circ} \mathrm{C}$ and washed with two consecutive solutions $\left(0.2 \% \mathrm{SDS}, 2 \mathrm{X} \mathrm{SSC}\right.$ at $42^{\circ} \mathrm{C}$ for $5 \mathrm{~min}$ and $0.2 \mathrm{X}$ $\mathrm{SSC}$ for $5 \mathrm{~min}$ at room temperature).

The array data were analyzed for data summarization, normalization and quality control using GeneSpring software
V12 (Agilent; Thermo Fisher Scientific, Inc.) (19). To select the differentially expressed genes (DEGs), threshold values of $\geq 2$ and $\leq-2$-fold change (FC) and a P-value of 0.05 were used. The data was $\log _{2}$-transformed and median centered by genes using the Adjust Data function of Cluster 3.0 software (www.falw.vu/ huik/cluster.htm), and then further analyzed by hierarchical clustering with average linkage (20). Finally, tree visualization was performed using Treeview (Stanford University School of Medicine, Stanford, CA, USA) (21).

Reverse transcription-quantitative polymerase chain reaction $(R T-q P C R)$ analysis. The DEGs regulated by leptin and hypoxia identified by the microarray, were verified using RT-qPCR analysis. In total, five genes [Arrestin $\beta 1$ (Arrb1), $\mathrm{C}-\mathrm{C}$ motif ligand $(\mathrm{Ccl}) 1, \mathrm{G}$ protein-coupled receptor kinase 4 (Grk)4, Ccl17 and C-C motif chemokine receptor 2 (Ccr2)] were selected. Total RNA was extracted and the quality was assessed, as described above. The first-strand cDNA was synthesized using $500 \mathrm{ng}$ total RNA in a $20.0 \mu \mathrm{l}$ final volume by reverse transcription utilizing PrimeScript ${ }^{\mathrm{TM}}$ RT Master mix (Perfect Real Time; Takara Bio, Inc., Otsu, Japan). Subsequently, the cDNA was diluted in five volumes sterile water. The qPCR was performed in a volume of $20.0 \mu \mathrm{l}$ using $2.0 \mu \mathrm{l}$ cDNA, $0.8 \mu 1$ specific forward primer, $0.8 \mu 1$ specific reverse primer, $10.0 \mu 1 \mathrm{SYBR}^{\circledR}$ Select Master mix (Thermo Fisher Scientific, USA) and $6.4 \mu 1$ deionized water. The amplification was performed using a Roche LightCycler ${ }^{\circledR}$ detection system (Roche Diagnostics, Indianapolis, IN, USA). The primers (Sangon Biotech Co, Ltd., Shanghai, China) were as follows: Arrb1, forward 5'-AGG CAT CAC TGG ATA AGG AG-3' and reverse 5'-GTC TTG TTG GTG TTG TTG GTG-3'; Ccl1, forward 5'-TTC CCC TGA AGT TTA TCC AG-3' and reverse 5'-GAT TTT GAA CCC ACG TTT TG-3'; Grk4, forward 5'-ATG GAG GGG ATT TGA AGT AC-3' and reverse 5'-CTG GCT TTA GGT CTC TGT AT-3'; Ccl17, forward 5'-GCT GCC TGG ATT ACT TCA AAG-3' and reverse 5'-TTT GTC TTT GGG GTC TGC AC-3'; Ccr2, forward 5'-TGT AGT CAC TTG GGT GGT GG-3' and reverse 5'-TAA GGG CCA CAG GTG TAA TG-3'. For all RT-qPCR experiments, negative controls comprised a non-reverse transcription reaction and a non-sample reaction (data not shown). Actin was amplified as an internal standard. The $2^{-\Delta \Delta \mathrm{Cq}}$ method was applied for data analysis (22).

Functional enrichment analysis. The Database for Annotation, Visualization, and Integrated Discovery (DAVID) is widely used in functional enrichment analysis of DEGs (23). In the present study, DAVID (david.abcc.ncifcrf.gov) was used to perform functional enrichment analysis for the DEGs regulated by leptin, hypoxia and the two combined, respectively. The genes were mapped to Gene Ontology (GO) terms for this purpose. The GO annotation (www.geneontology.org) provides a descriptive framework and functional annotation of DEGs, and is comprised of biological processes, cellular components and molecular functions. In addition, Kyoto Encyclopedia of Genes and Genomes (KEGG; http://www.genome.jp/kegg/) pathway enrichment analysis was performed to map the potential pathways of the DEGs (24). The P-value cut-off associated with this analysis was set at $\mathrm{P}<0.05$ in order to identify significantly enriched functional terms and pathways. 
Cell cycle analysis using flow cytometry (FCM). The cells were seeded at a density of $10 \times 10^{4}$ per well in six-well plates in triplicate and allowed to adhere for $24 \mathrm{~h}$. Following starvation, the cells were treated with or without leptin $(200 \mathrm{ng} / \mathrm{ml})$ in normoxic conditions at $37^{\circ} \mathrm{C}$. Following culture for $24 \mathrm{~h}$, the cells were harvested and fixed in $70 \%$ cold ethanol at $-20^{\circ} \mathrm{C}$ overnight. The cells were stained with propidium iodide (Sigma-Aldrich; Merck Millipore) at $50 \mu \mathrm{g} / \mathrm{ml}$ with $20 \mu \mathrm{g} / \mathrm{ml}$ RNase A at room temperature in the dark for $1 \mathrm{~h}$ prior to analysis. The cell population fraction in each phase of the cell cycle was determined as a function of the DNA content using FCM (FACSCalibur; BD Biosciences, Franklin Lakes, NJ, USA). Data analysis was performed using FlowJo v10 software (Tree Star, Inc., Ashland, OR, USA) (25). This experiment was repeated three times.

Statistical analysis. Values are presented as the mean \pm standard deviation unless otherwise indicated using SPSS version 13.0 (SPSS, Inc., Chicago, IL, USA). Statistical analysis was performed using Student's t-test. $\mathrm{P}<0.05$ was considered to indicate a statistically significant difference.

\section{Results}

Microarray analysis and hierarchical clustering. The genes induced in the cultured L929 cells by leptin, hypoxia and the two combined were analyzed using a cDNA array. The array included four groups containing 12 samples. The cluster results of the four sets of microarray data are shown in Fig. 1. The two primary gene clusters were identified visually based on the heat map signal intensity in groups I and II, vs. groups III and IV. The expression of genes in cluster 1 were higher in groups I and II, compared with that in groups III and IV, suggesting that those genes may be suppressed by hypoxia. By contrast, cluster 2 consisted of genes activated by hypoxia. It appeared that leptin was a weak factor affecting gene profiling in normoxia and hypoxia.

The genes with $\mathrm{FC}$ values $>2.0$ and $\mathrm{P}<0.05$ were considered to be a DEG. In the present study, 54 DEGs were found in the leptin-treated group, of which 52 (96.30\%) were downregulated. A total of 1,507 DEGs were found under hypoxia treatment, of which 467 (30.99\%) were downregulated. In the group treated with leptin and hypoxia, 1,502 DEGs were found, among which $495(32.96 \%)$ were downregulated. However, compared with the hypoxia group, there were only 11 genes altered in the leptin and hypoxia treatment group, comprising three $(27.27 \%)$ downregulated and eight $(72.73 \%)$ upregulated genes.

Verification of array data using $R T-q P C R$ analysis. To assess the reliability of the array data, five genes (Arrb1, Ccl1, Grk4, Ccl17 and Ccr2) were selected for amplification, with normoxia and hypoxia samples as a template, using RT-qPCR analysis. The $2^{-\Delta \Delta \mathrm{Cq}}$ method was used for the determination of target mRNA following normalizing of target mRNA Cq values with those for actin $(\Delta \mathrm{Cq})$. In the array data, two genes (Arrb1 and Ccl1) were upregulated in the hypoxia samples by 3.51- and 6.78 -fold, respectively. In the RT-qPCR experiments, the upregulated FC values were 2.78- and 5.44-fold, respectively (Fig. 2). The other three genes (Grk4, Ccl17 and Ccr2) were downregulated in the hypoxia samples, by

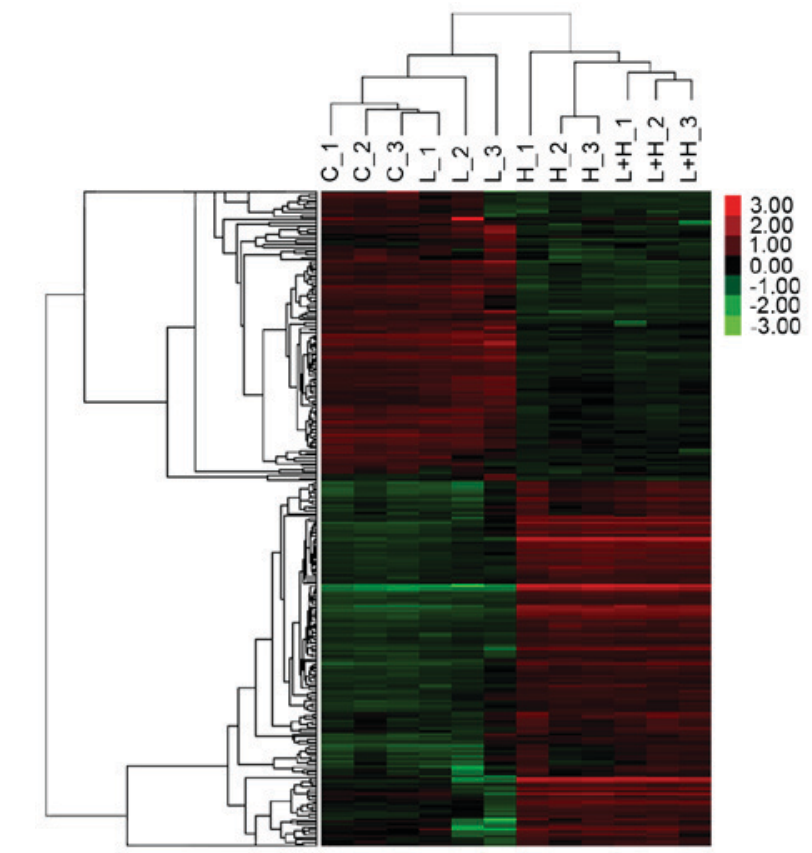

Figure 1. Array data. Hierarchical clustering dendrogram comparing leptin-altered, hypoxia-altered, and leptin and hypoxia-treated groups to normoxia control exposure. Each sample listed contains the average gene expression value for three replicates. C, normoxia control; L, leptin $200 \mathrm{ng} / \mathrm{ml}$; H, hypoxia, $1 \% \mathrm{O} 2$; L+H, leptin and hypoxia (200 ng/ml leptin and $1 \% \mathrm{O} 2$ ). Green, low expression; red, high expression.

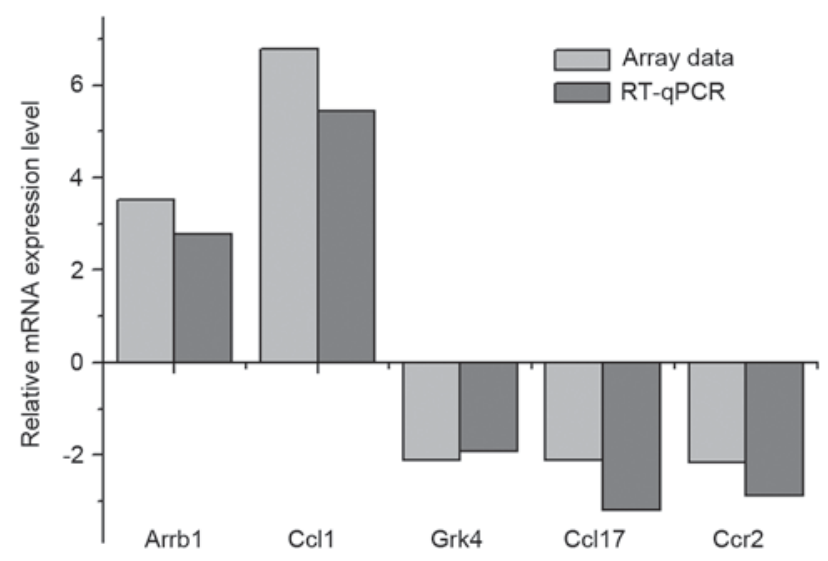

Figure 2. RT-qPCR verification of the array data. Fold changes of selected genes in normoxia and hypoxia groups using microarray and RT-qPCR analyses. RT-qPCR, reverse transcription-quantitative polymerase chain reaction; Arrb1, Arrestin $\beta 1$; Ccl1, C-C motif ligand 1, Grk4, G protein-coupled receptor kinase 4; Ccl17, C-C motif ligand 17; Ccr2, C-C motif chemokine receptor 2 .

2.11-, 2.11- and 2.15-fold, respectively, and the FC values in the RT-qPCR experiments were 1.92-, 3.19- and 2.87-fold, respectively (Fig. 2). These results suggested that the array data was in correspondence with the RT-qPCR experiments.

Functional enrichment analysis. To investigate the biological roles of the DEGs regulated by leptin, hypoxia and the two combined in L929 cells, a categorized GO enrichment analysis was performed, comprising 54, 1,507 and 1,502 genes, respectively (Tables I-III). For the DEGs response to leptin, meiosis 

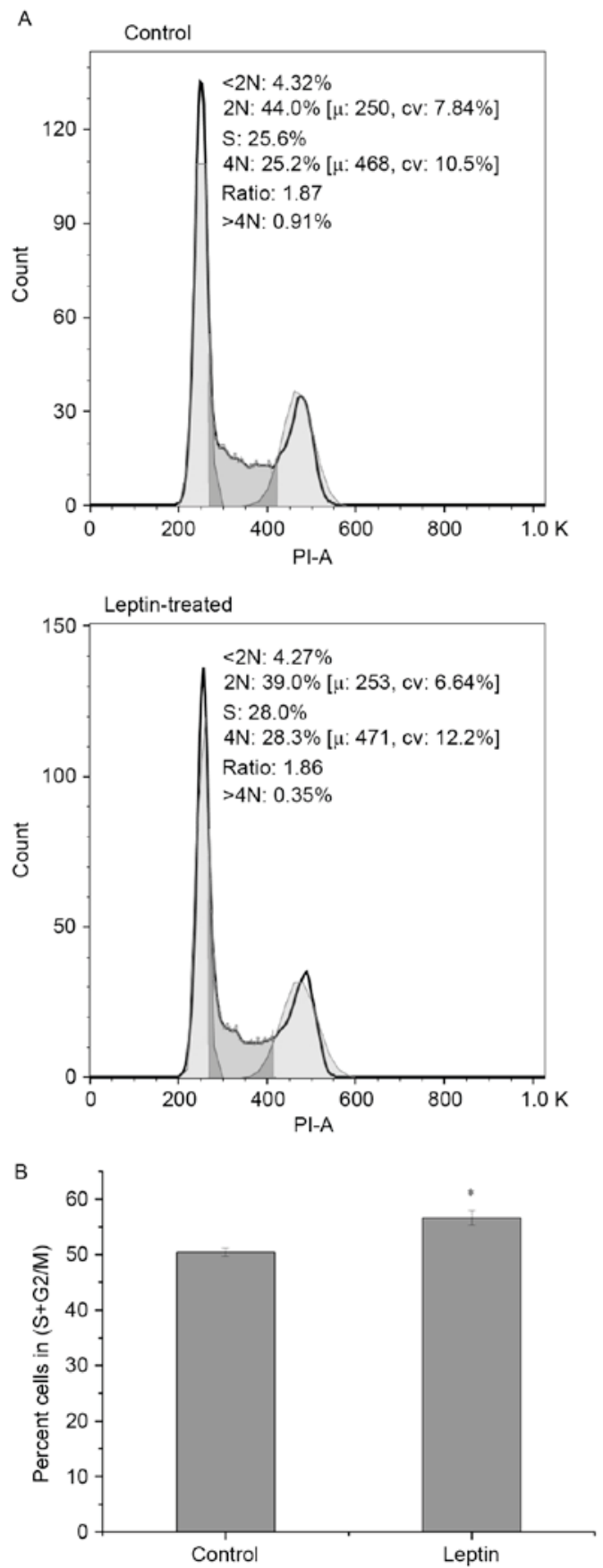

Figure 3. Effects of leptin on cell cycle progression in L929 cells. The cells were treated with $200 \mathrm{ng} / \mathrm{ml}$ leptin for $24 \mathrm{~h}$, and (A) DNA content was analyzed using fluorescence flow cytometry following PI staining. (B) Percentages of cells in the $\mathrm{S}+\mathrm{G} 2 / \mathrm{M}$ phase. $\mathrm{PI}$, propidium iodide. ${ }^{*} \mathrm{P}=0.033$.

I $(\mathrm{P}=0.004)$ and synaptonemal complex $(\mathrm{P}=0.001)$ were the most significantly enriched functional terms for biological processes and cellular components, respectively. For the DEGs response to hypoxia, glucose metabolic process $(\mathrm{P}=0.0008)$, cell projection part $(\mathrm{P}=0.003)$ and ion binding $(\mathrm{P}=7.8621 \mathrm{E}-05)$ were the most significantly enriched functional terms for biological processes, cellular components and molecular functions, respectively. For the DEGs regulated by leptin and hypoxia combined, phosphate metabolic process $(\mathrm{P}=0.0007)$ and extracellular region $(\mathrm{P}=0.0022)$ were the most significantly enriched functional terms for biological processes and cellular components, respectively.
Table I. GO analysis for the differentially expressed genes regulated by leptin.

\begin{tabular}{|c|c|c|}
\hline Term & Genes (n) & P-value \\
\hline Molecular function & - & - \\
\hline \multicolumn{3}{|l|}{ Biological process } \\
\hline GO:0007127 meiosis I & 3 & 0.004056677 \\
\hline $\begin{array}{l}\text { GO: } 0022402 \text { cell } \\
\text { cycle process }\end{array}$ & 5 & 0.021448529 \\
\hline $\begin{array}{l}\text { GO: } 0051327 \mathrm{M} \text { phase of } \\
\text { meiotic cell cycle }\end{array}$ & 3 & 0.023847468 \\
\hline GO:0007126 meiosis & 3 & 0.023847468 \\
\hline GO:0007049 cell cycle & 6 & 0.023981901 \\
\hline GO:0051321 meiotic cell cycle & 3 & 0.024865727 \\
\hline GO:0007129 synapsis & 2 & 0.045322159 \\
\hline $\begin{array}{l}\text { GO:0070192 chromosome } \\
\text { organization involved in meiosis }\end{array}$ & 2 & 0.045322159 \\
\hline \multicolumn{3}{|l|}{ Cell component } \\
\hline $\begin{array}{l}\text { GO:0000795 synaptonemal } \\
\text { complex }\end{array}$ & 3 & 0.001322361 \\
\hline $\begin{array}{l}\text { GO:0044454 nuclear } \\
\text { chromosome part }\end{array}$ & 4 & 0.002698438 \\
\hline $\begin{array}{l}\text { GO:0000793 condensed } \\
\text { chromosome }\end{array}$ & 4 & 0.003006214 \\
\hline $\begin{array}{l}\text { GO:0000228 nuclear } \\
\text { chromosome }\end{array}$ & 4 & 0.004250276 \\
\hline $\begin{array}{l}\text { GO:0000794 condensed } \\
\text { nuclear chromosome }\end{array}$ & 3 & 0.005772372 \\
\hline GO:0044427 chromosomal part & 5 & 0.010435104 \\
\hline GO:0005694 chromosome & 5 & 0.018637296 \\
\hline GO:0000800 lateral element & 2 & 0.018883838 \\
\hline
\end{tabular}

GO, Gene Ontology.

Pathway enrichment analysis. KEGG pathway enrichment analysis was performed to assess the biological roles of the DEGs (Table IV). Axon guidance was the only significant pathway in response to leptin $(\mathrm{P}=0.0294)$. There were 12 significant pathways in response to hypoxia, among which the chemokine signaling pathway $(\mathrm{P}=0.00014)$ was the most significant, which suggested inflammatory factors were crucial in the response to hypoxia in L929 cells. For the combined treatment group, nine pathways were significant, of which eight were identical to the responses to hypoxia: Nitrogen metabolism, focal adhesion, chemokine signaling pathway, arginine and proline metabolism, starch and sucrose metabolism, pyruvate metabolism, VEGF signaling pathway and MAPK signaling pathway.

Leptin promotes the proliferation of L929 cells. The results of the functional enrichment analysis suggested that leptin affected the cell cycle progression of L929 cells under hypoxia. To confirm this, the numbers of cells in different cell cycle phases were detected using FCM. Exposing the L929 cells to leptin resulted in a high percentage of cells in the $\mathrm{S}+\mathrm{G} 2 / \mathrm{M}$ 
Table II. GO analysis for the differentially expressed genes regulated by hypoxia.

\begin{tabular}{|c|c|c|}
\hline Term & Genes (n) & $\mathrm{P}$-value \\
\hline \multicolumn{3}{|l|}{ Molecular function } \\
\hline GO:0043167 ion binding & 333 & $7.8621 \mathrm{E}-05$ \\
\hline GO:0043169 cation binding & 329 & 8.79225E-05 \\
\hline GO:0046872 metal ion binding & 326 & 9.99094E-05 \\
\hline GO:0016836 hydro-lyase activity & 12 & 0.000122629 \\
\hline GO:0017016 Ras GTPase binding & 12 & 0.001984074 \\
\hline GO:0000287 magnesium ion binding & 46 & 0.002246305 \\
\hline GO:0031267 small GTPase binding & 12 & 0.002642921 \\
\hline \multicolumn{3}{|c|}{ GO:0016702 oxidoreductase activity, acting on single donors with incorporation } \\
\hline of molecular oxygen, incorporation of two atoms of oxygen & 13 & 0.002791662 \\
\hline \multicolumn{3}{|l|}{ GO:0016701 oxidoreductase activity, acting on single donors with } \\
\hline GO:0051020 GTPase binding & 12 & 0.003951419 \\
\hline GO:0046914 transition metal ion binding & 218 & 0.005063268 \\
\hline GO:0019899 enzyme binding & 28 & 0.006555908 \\
\hline GO:0005506 iron ion binding & 38 & 0.007222115 \\
\hline GO:0001882 nucleoside binding & 135 & 0.009641824 \\
\hline GO:0001883 purine nucleoside binding & 134 & 0.010743224 \\
\hline GO:0031418 L-ascorbic acid binding & 6 & 0.011357513 \\
\hline GO:0004725 protein tyrosine phosphatase activity & 15 & 0.011975484 \\
\hline GO:0030554 adenyl nucleotide binding & 132 & 0.01339165 \\
\hline GO:0003779 actin binding & 32 & 0.013513591 \\
\hline GO:0004674 protein serine/threonine kinase activity & 43 & 0.016010492 \\
\hline GO:0031406 carboxylic acid binding & 13 & 0.016733784 \\
\hline GO:0004672 protein kinase activity & 56 & 0.018393253 \\
\hline GO:0008092 cytoskeletal protein binding & 42 & 0.019021647 \\
\hline GO:0005524 ATP binding & 123 & 0.022617503 \\
\hline GO:0019992 diacylglycerol binding & 10 & 0.022656776 \\
\hline GO:0019842 vitamin binding & 16 & 0.024555951 \\
\hline GO:0015293 symporter activity & 17 & 0.02501501 \\
\hline GO:0017076 purine nucleotide binding & 154 & 0.029861653 \\
\hline GO:0032559 adenyl ribonucleotide binding & 123 & 0.030297935 \\
\hline \multicolumn{3}{|l|}{ GO:0016641 oxidoreductase activity, acting on the $\mathrm{CH}-\mathrm{NH} 2$ group of } \\
\hline GO:0004089 carbonate dehydratase activity & 5 & 0.034869252 \\
\hline GO:0050662 coenzyme binding & 19 & 0.035408286 \\
\hline GO:0004721 phosphoprotein phosphatase activity & 18 & 0.042027399 \\
\hline \multicolumn{3}{|l|}{ GO:0016723 oxidoreductase activity, oxidizing metal ions, NAD or NADP } \\
\hline as acceptor & 3 & 0.043728093 \\
\hline GO:0008138 protein tyrosine/serine/threonine phosphatase activity & 7 & 0.049875355 \\
\hline \multicolumn{3}{|l|}{ Biological process } \\
\hline GO:0006006 glucose metabolic process & 22 & 0.000792317 \\
\hline GO:0055114 oxidation reduction & 70 & 0.000950229 \\
\hline GO:0006796 phosphate metabolic process & 83 & 0.003423909 \\
\hline GO:0006793 phosphorus metabolic process & 83 & 0.003423909 \\
\hline GO:0044271 nitrogen compound biosynthetic process & 35 & 0.004517996 \\
\hline GO:0007601 visual perception & 16 & 0.004646543 \\
\hline GO:0050953 sensory perception of light stimulus & 16 & 0.00510266 \\
\hline GO:0007242 intracellular signaling cascade & 85 & 0.006819851 \\
\hline GO:0019318 hexose metabolic process & 22 & 0.007922216 \\
\hline GO:0005996 monosaccharide metabolic process & 24 & 0.008183748 \\
\hline GO:0044275 cellular carbohydrate catabolic process & 11 & 0.008669622 \\
\hline
\end{tabular}


Table II. Continued.

\begin{tabular}{|c|c|c|}
\hline Term & Genes (n) & P-value \\
\hline GO:0006468 protein amino acid phosphorylation & 62 & 0.009474011 \\
\hline GO:0006096 glycolysis & 9 & 0.010826957 \\
\hline GO:0008015 blood circulation & 16 & 0.011053616 \\
\hline GO:0003013 circulatory system process & 16 & 0.011053616 \\
\hline GO:0001666 response to hypoxia & 11 & 0.013524164 \\
\hline GO:0070482 response to oxygen levels & 11 & 0.015005602 \\
\hline GO:0042384 cilium assembly & 6 & 0.016566907 \\
\hline GO:0007010 cytoskeleton organization & 34 & 0.022567205 \\
\hline GO:0051241 negative regulation of multicellular organismal process & 14 & 0.023152487 \\
\hline GO:0030029 actin filament-based process & 21 & 0.0232384 \\
\hline GO:0030036 actin cytoskeleton organization & 20 & 0.023432887 \\
\hline GO:0048545 response to steroid hormone stimulus & 10 & 0.023828973 \\
\hline GO:0019374 galactolipid metabolic process & 3 & 0.02707365 \\
\hline GO:0006681 galactosylceramide metabolic process & 3 & 0.02707365 \\
\hline GO:0019320 hexose catabolic process & 9 & 0.028066811 \\
\hline GO:0006007 glucose catabolic process & 9 & 0.028066811 \\
\hline GO:0006470 protein amino acid dephosphorylation & 15 & 0.029397775 \\
\hline GO:0006767 water-soluble vitamin metabolic process & 7 & 0.029717956 \\
\hline GO:0051046 regulation of secretion & 16 & 0.029739379 \\
\hline GO:0016310 phosphorylation & 65 & 0.030184101 \\
\hline GO:0016265 death & 49 & 0.03112936 \\
\hline GO:0060271 cilium morphogenesis & 6 & 0.032808209 \\
\hline GO:0009743 response to carbohydrate stimulus & 6 & 0.032808209 \\
\hline GO:0006778 porphyrin metabolic process & 6 & 0.032808209 \\
\hline GO:0033013 tetrapyrrole metabolic process & 6 & 0.032808209 \\
\hline GO:0009967 positive regulation of signal transduction & 20 & 0.034149817 \\
\hline GO:0046365 monosaccharide catabolic process & 9 & 0.034329668 \\
\hline GO:0060341 regulation of cellular localization & 19 & 0.034742883 \\
\hline GO:0009719 response to endogenous stimulus & 21 & 0.035172676 \\
\hline GO:0006730 one-carbon metabolic process & 15 & 0.035666401 \\
\hline GO:0042403 thyroid hormone metabolic process & 4 & 0.037600598 \\
\hline GO:0046164 alcohol catabolic process & 10 & 0.03779069 \\
\hline GO:0016311 dephosphorylation & 17 & 0.039806519 \\
\hline GO:0006357 regulation of transcription from RNA polymerase II promoter & 56 & 0.04088076 \\
\hline GO:0008219 cell death & 47 & 0.046075704 \\
\hline GO:0006955 immune response & 44 & 0.047117585 \\
\hline GO:0048660 regulation of smooth muscle cell proliferation & 5 & 0.04805859 \\
\hline GO:0050873 brown fat cell differentiation & 6 & 0.049834676 \\
\hline \multicolumn{3}{|l|}{ Cell component } \\
\hline GO:0044463 cell projection part & 23 & 0.003220028 \\
\hline GO:0042995 cell projection & 58 & 0.003517983 \\
\hline GO:0005886 plasma membrane & 234 & 0.004494904 \\
\hline GO:0005576 extracellular region & 143 & 0.00502389 \\
\hline GO:0031225 anchored to membrane & 25 & 0.008680624 \\
\hline GO:0019898 extrinsic to membrane & 46 & 0.017185716 \\
\hline GO:0044441 cilium part & 8 & 0.020951644 \\
\hline GO:0042579 microbody & 14 & 0.026699591 \\
\hline GO:0005777 peroxisome & 14 & 0.026699591 \\
\hline GO:0044421 extracellular region part & 68 & 0.031279026 \\
\hline GO:0045121 membrane raft & 12 & 0.031670056 \\
\hline GO:0043005 neuron projection & 26 & 0.032261958 \\
\hline GO:0019897 extrinsic to plasma membrane & 9 & 0.034092369 \\
\hline
\end{tabular}


Table II. Continued.

\begin{tabular}{lcr}
\hline Term & Genes (n) & P-value \\
\hline GO:0005829 cytosol & 50 & 0.038894078 \\
GO:0005930 axoneme & 7 & 0.039557367 \\
GO:0005901 caveola & 6 & 0.04056632 \\
GO:0031672 A band & 4 & 0.045209466 \\
GO:0005929 cilium & 16 & 0.045846025 \\
GO:0042598 vesicular fraction & 20 & 0.046732261 \\
GO:0005741 mitochondrial outer membrane & 11 & 0.048199094 \\
GO:0030016 myofibril & 12 & 0.048557001 \\
\hline
\end{tabular}

GO, Gene Ontology.

Table III. GO analysis for the differentially expressed genes regulated by leptin and hypoxia.

Term $\quad$ Genes (n) P-value

Molecular function

Biological process

GO:0006796 phosphate metabolic process

GO:0006793 phosphorus metabolic process

GO:0016265 death

GO:0006468 protein amino acid phosphorylation

GO:0017157 regulation of exocytosis

GO:0008219 cell death

GO:0055114 oxidation reduction

GO:0012501 programmed cell death

GO:0044271 nitrogen compound biosynthetic process

GO:0006915 apoptosis

GO:0016310 phosphorylation

GO:0003016 respiratory system process

GO:0009743 response to carbohydrate stimulus

GO:0006006 glucose metabolic process

GO:0051241 negative regulation of multicellular organismal process

GO:0007601 visual perception

GO:0032940 secretion by cell

GO:0050953 sensory perception of light stimulus

GO:0046903 secretion

GO:0001666 response to hypoxia

GO:0070482 response to oxygen levels

GO:0009746 response to hexose stimulus

GO:0001974 blood vessel remodeling

GO:0009749 response to glucose stimulus

GO:0034284 response to monosaccharide stimulus

GO:0006730 one-carbon metabolic process

GO:0009719 response to endogenous stimulus

GO:0048608 reproductive structure development

GO:0048545 response to steroid hormone stimulus

GO:0003013 circulatory system process

GO:0008015 blood circulation

GO:0001775 cell activation

GO:0007242 intracellular signaling cascade

GO:0006865 amino acid transport
0.00069201

0.00069201

0.000914867

0.001122958

0.001368273

0.001517519

0.002418208

0.002614104

0.004597316

0.004983028

0.005034959

0.005961782

0.008246776

0.009614291

0.010348645

0.011259001

0.011798862

0.012230633

0.013035463

0.013616099

0.015106629

0.016635896

0.016635896

0.016635896

0.016635896

0.01753354

0.019740633

0.020659779

0.023972008

0.024166653

0.024166653

0.025299039

0.02535934

0.026707116 
Table III. Continued.

\begin{tabular}{|c|c|c|}
\hline Term & Genes (n) & P-value \\
\hline GO:0006681 galactosylceramide metabolic process & 3 & 0.027127455 \\
\hline GO:0019374 galactolipid metabolic process & 3 & 0.027127455 \\
\hline GO:0005996 monosaccharide metabolic process & 22 & 0.028503233 \\
\hline GO:0019318 hexose metabolic process & 20 & 0.029456322 \\
\hline GO:0006470 protein amino acid dephosphorylation & 15 & 0.029624933 \\
\hline \multicolumn{3}{|l|}{ GO:0045944 positive regulation of transcription from RNA } \\
\hline polymerase II promoter & 36 & 0.030944318 \\
\hline GO:0006778 porphyrin metabolic process & 6 & 0.032936937 \\
\hline GO:0033013 tetrapyrrole metabolic process & 6 & 0.032936937 \\
\hline GO:0003006 reproductive developmental process & 28 & 0.033137813 \\
\hline GO:0003001 generation of a signal involved in cell-cell signaling & 12 & 0.033562678 \\
\hline GO:0009967 positive regulation of signal transduction & 20 & 0.034460687 \\
\hline GO:0046324 regulation of glucose import & 5 & 0.040773001 \\
\hline \multicolumn{3}{|l|}{ GO:0006357 regulation of transcription from RNA polymerase II } \\
\hline promoter & 56 & 0.041404785 \\
\hline GO:0045893 positive regulation of transcription, DNA-dependent & 40 & 0.041795439 \\
\hline GO:0006979 response to oxidative stress & 12 & 0.041889132 \\
\hline GO:0051254 positive regulation of RNA metabolic process & 40 & 0.045730238 \\
\hline GO:0009220 pyrimidine ribonucleotide biosynthetic process & 4 & 0.047696549 \\
\hline GO:0009218 pyrimidine ribonucleotide metabolic process & 4 & 0.047696549 \\
\hline GO:0010827 regulation of glucose transport & 5 & 0.048213767 \\
\hline GO:0042398 cellular amino acid derivative biosynthetic process & 8 & 0.049928789 \\
\hline \multicolumn{3}{|l|}{ Cell component } \\
\hline GO:0005576 extracellular region & 145 & 0.002250808 \\
\hline GO:0044463 cell projection part & 23 & 0.003001467 \\
\hline GO:0005777 peroxisome & 16 & 0.00482117 \\
\hline GO:0042579 microbody & 16 & 0.00482117 \\
\hline GO:0042995 cell projection & 55 & 0.011330557 \\
\hline GO:0045121 membrane raft & 13 & 0.013007776 \\
\hline GO:0031225 anchored to membrane & 24 & 0.015194373 \\
\hline GO:0005886 plasma membrane & 227 & 0.016114278 \\
\hline GO:0008021 synaptic vesicle & 11 & 0.017112432 \\
\hline GO:0044456 synapse part & 24 & 0.019656437 \\
\hline GO:0033267 axon part & 6 & 0.025413 \\
\hline GO:0044421 extracellular region part & 68 & 0.028509841 \\
\hline GO:0019898 extrinsic to membrane & 44 & 0.035509102 \\
\hline GO:0005730 nucleolus & 31 & 0.035643165 \\
\hline GO:0043232 intracellular non-membrane-bounded organelle & 152 & 0.036036538 \\
\hline GO:0043228 non-membrane-bounded organelle & 152 & 0.036036538 \\
\hline
\end{tabular}

GO, Gene Ontology.

phases, which indicated that leptin promoted L929 cell proliferation (Fig. 3A and B).

\section{Discussion}

Investigations have increasingly focused on the pro-fibrotic microenvironment of organs. Leptin and hypoxia are pro-fibrotic factors, which are involved in fibrogenesis. In the present study, a high-throughput microarray method was applied to detect the expression profile response to leptin, hypoxia and the two combined in L929 cells. It was found that leptin promoted mouse fibroblast cell proliferation, whereas hypoxia affected L929 cell function, primarily through the chemokine signaling pathway.

The present study identified 54 leptin-responsive genes, 52 of which were downregulated $>2$-fold. Among these, nephronophthisis 3, also known as pcy, showed a marked reduction by 3.3 -fold. It has been reported that pcy mice undergoing 
Table IV. Pathway analysis of the differentially expressed genes regulated by leptin, hypoxia and the two in combination.

\begin{tabular}{|c|c|c|c|}
\hline KEGG ID & Term & $\mathrm{n}$ & P-value \\
\hline $\begin{array}{c}\text { Leptin-treated } \\
\text { mmu04360 }\end{array}$ & \multicolumn{2}{|c|}{ Leptin-treated } & 0.029392665 \\
\hline \multicolumn{4}{|c|}{ Hypoxia-treated } \\
\hline mmu04062 & Chemokine signaling pathway & 28 & 0.00014053 \\
\hline mmu00500 & Starch and sucrose metabolism & 10 & 0.000638251 \\
\hline mmu00910 & Nitrogen metabolism & 7 & 0.004127542 \\
\hline mmu00052 & Galactose metabolism & 7 & 0.009539235 \\
\hline mmu04510 & Focal adhesion & 24 & 0.010772098 \\
\hline mmu00380 & Tryptophan metabolism & 8 & 0.019533919 \\
\hline mmu04360 & Axon guidance & 17 & 0.020000767 \\
\hline mmu00620 & Pyruvate metabolism & 8 & 0.022185656 \\
\hline mmu00330 & Arginine and proline metabolism & 9 & 0.0297123 \\
\hline mmu04010 & MAPK signaling pathway & 28 & 0.029963278 \\
\hline mmu04370 & VEGF signaling pathway & 11 & 0.03808443 \\
\hline mmu00010 & Glycolysis/gluconeogenesis & 10 & 0.046234215 \\
\hline \multicolumn{4}{|c|}{ Leptin and hypoxia-treated } \\
\hline mmu00910 & Nitrogen metabolism & 7 & 0.004076684 \\
\hline mmu04510 & Focal adhesion & 25 & 0.005345589 \\
\hline $\mathrm{mmu04062}$ & Chemokine signaling pathway & 23 & 0.007744979 \\
\hline mmu00330 & Arginine and proline metabolism & 10 & 0.010268331 \\
\hline mmu00500 & Starch and sucrose metabolism & 8 & 0.010980612 \\
\hline mmu00620 & Pyruvate metabolism & 8 & 0.021915205 \\
\hline mmu04630 & JAK-STAT signaling pathway & 18 & 0.035405855 \\
\hline mmu04370 & VEGF signaling pathway & 11 & 0.037534097 \\
\hline mmu04010 & MAPK signaling pathway & 27 & 0.047450835 \\
\hline
\end{tabular}

KEGG, Kyoto Encyclopedia of Genes and Genomes; MAPK, mitogen-activated protein kinase; VEGF, vascular endothelial growth factor; JAK, Janus kinase; STAT, signal transducer and activator of transcription.

cystogenesis present with progressive increasingly severe renal fibrosis (26). Another gene, E2f transcription factor 4 (E2f4), showed a marked reduction by 3.0 -fold. E2f4 is important in the suppression of proliferation-associated genes and is involved in the G1/S phase of the mitotic cell cycle (27-30). This may account for the percentage of cells in the G1/S phase being decreased and that in the $\mathrm{S}+\mathrm{G} 2 / \mathrm{M}$ being increased in response to leptin. Leptin-stimulated cell proliferation had been reported previously, including in vascular smooth muscle cell proliferation (31), hepatic stellate cells (32) and cancer cells (33).

Pathway analysis revealed the significant pathway regulated by leptin was axon guidance, of which three genes, Eph receptor A5, Rho-associated coiled-coil containing protein kinase 1 (Rock1) and semaphoring 6D, were significantly affected. These results were concordant with previous studies. A study by Simerly (34) found that leptin may direct the development of hypothalamic pathways by promoting axonal projections. A study by Harrold (35) indicated novel regulatory roles for leptin in synaptic plasticity and axon guidance.

Several genes varied in response to hypoxia. The most significant pathway response to hypoxia was the chemokine signaling pathway, and the expression of 28 genes (Ccl1, adenylate cyclase 4 , protein kinase $C, G$ protein subunit $\alpha 1, \mathrm{Cxc19}, \mathrm{G}$ protein subunit $\gamma$ (Gng)13, Cxcl10, dedicator of cytokinesis 2, son of sevenless homolog 1, Gng2, phosphoinositide-3-kinase regulatory subunit 3, phospholipase $\mathrm{C} \beta 2$, SHC adaptor protein 2, AKT serine/threonine kinase 2, Gng7, mitogen-activated protein kinase kinase 1, Rock1, vav guanine nucleotide exchange factor $1, \mathrm{Ccl17}$, engulfment and cell motility 1, Arrb1, glycogen synthase kinase $3 \beta$, Ccr2, G protein subunit $\beta 5$, RAP1A, member of RAS oncogene family, Grk4, Jak3 and Crk) were altered in this pathway. Among these, $\mathrm{Ccr} 2$ and $\mathrm{CC}$ chemokine ligand $2(\mathrm{Ccl} 2)$ receptor were previously reported to be altered in oxygen shortage (36). In addition, $\mathrm{Cxc19}, \mathrm{Cxcl10}$ and $\mathrm{Ccl} 17$ have been reported to be involved in the pathogenesis of lung fibrosis (37). This result further suggested that inflammation was important in L929 function, particularly in pathological states. Therefore, it was hypothesized that the hypoxic microenvironment facilitates L929 cell proliferation through the chemokine signaling pathway, and the uncontrolled inflammation further promotes fibrosis. Further understanding of the mechanisms involved in chemokine-mediated cell proliferation may lead to improved therapeutic strategies in fibrosis.

Several other pathways were involved in the response to hypoxia, including starch and sucrose metabolism, nitrogen 
metabolism and galactose metabolism. These pathways associated to metabolism were in accordance with expectations, as cell adaptation to low oxygen concentrations involves repression of mitochondrial respiration and induction of glycolysis to sustain cell function in hypoxic conditions (38). Axon guidance was also a significant pathway response to hypoxia and to leptin, which was coincident with a previous study (39). Therefore, under hypoxia, several pathways may function in concert to restore oxygen supply to cells and modulate cell function to adapt the hypoxic conditions.

In conclusion, the present study showed that leptin and hypoxia altered gene expression profiles in L929 cells. The results suggested that the pro-fibrotic effects of leptin may be through promoting mouse fibroblast cell proliferation; whereas hypoxia affected mouse fibroblast cell function predominantly through the chemokine signaling pathway. These findings improve understanding of leptin and hypoxia in fibroblast cells. Axon guidance and the chemokine signaling pathway may represent novel therapeutic targets for leptin and hypoxia injury in fibrogenesis, and require further investigation.

\section{Acknowledgements}

This study was supported by grants from the National Natural Science Foundation of China (grant nos. 81200082, 81302244 and 81502899), the Medical Science and Technology Research Fund of Guangdong province (grant no. B2012272) and the PhD Start-up Fund of Guangdong Medical College (grant no. B2011019).

\section{References}

1. Sziksz E, Pap D, Lippai R, Béres NJ, Fekete A, Szabó AJ and Vannay A: Fibrosis related inflammatory mediators: Role of the IL-10 cytokine family. Mediators Inflamm 2015: 764641, 2015.

2. Tacke F and Trautwein C: Mechanisms of liver fibrosis resolution. J Hepatol 63: 1038-1039, 2015.

3. Wynn TA: Cellular and molecular mechanisms of fibrosis. J Pathol 214: 199-210, 2008.

4. Peters S: Cystic fibrosis: A review of pathophysiology and current treatment recommendations. S D Med 67: 148-151, 153, 2014.

5. Tao H, Shi KH, Yang JJ, Huang C, Liu LP and Li J: Epigenetic regulation of cardiac fibrosis. Cell Signal 25: 1932-1938, 2013.

6. Uhal BD: Epithelial apoptosis in the initiation of lung fibrosis. Eur Respir J Suppl 44: S7-S9, 2003.

7. Friedman SL: Hepatic fibrosis-overview. Toxicology 254: 120-129, 2008

8. Zhan L, Huang C, Meng XM, Song Y, Wu XQ, Yang Y and Li J: Hypoxia-inducible factor-1alpha in hepatic fibrosis: A promising therapeutic target. Biochimie 108: 1-7, 2015.

9. Lokmic Z, Musyoka J, Hewitson TD and Darby IA: Hypoxia and hypoxia signaling in tissue repair and fibrosis. Int Rev Cell Mol Biol 296: 139-185, 2012.

10. Martínez-Martínez E, Jurado-López R, Valero-Muñoz M, Bartolomé MV, Ballesteros S, Luaces M, Briones AM, López-Andrés N, Miana M and Cachofeiro V: Leptin induces cardiac fibrosis through galectin-3, mTOR and oxidative stress: Potential role in obesity. J Hypertens 32: 1104-1114, 2014.

11. Handy JA, Fu PP, Kumar P, Mells JE, Sharma S, Saxena NK and Anania FA: Adiponectin inhibits leptin signalling via multiple mechanisms to exert protective effects against hepatic fibrosis. Biochem J 440: 385-395, 2011.

12. Chuang JH, Wang PW and Tai MH: An adipocentric view of liver fibrosis and cirrhosis. Chang Gung Med J 27: 855-868, 2004.

13. Zibadi S, Cordova F, Slack EH, Watson RR and Larson DF: Leptin's regulation of obesity-induced cardiac extracellular matrix remodeling. Cardiovasc Toxicol 11: 325-333, 2011.

14. Koyama Y and Brenner DA: New therapies for hepatic fibrosis. Clin Res Hepatol Gastroenterol 39 (Suppl 1): S75-S79, 2015.
15. Xie S, Chen H, Li F, Wang S and Guo J: Hypoxia-induced microRNA-155 promotes fibrosis in proximal tubule cells. Mol Med Rep 11: 4555-4560, 2015.

16. Tang J, Jiang X, Zhou Y and Dai Y: Effects of A2BR on the biological behavior of mouse renal fibroblasts during hypoxia. Mol Med Rep 11: 4397-4402, 2015.

17. Fan GP, Wang W, Zhao H, Cai L, Zhang PD, Yang ZH, Zhang J and Wang X: Pharmacological inhibition of focal adhesion kinase attenuates cardiac fibrosis in mice cardiac fibroblast and post-myocardial-infarction models. Cell Physiol Biochem 37: 515-526, 2015.

18. Melo NC, Amorim FF and Santana AN: Connecting the dots: Hypoxia, pulmonary fibrosis, obstructive sleep apnea, and aging. Am J Respir Crit Care Med 191: 966, 2015.

19. Koyama D, Maruoka S, Gon Y, Shintani Y, Sekiyama T, Hiranuma H, Shikano S, Kuroda K, Takeshita I, Tsuboi E, et al: Myeloid differentiation-2 is a potential biomarker for the amplification process of allergic airway sensitization in mice. Allergol Int 64 (Suppl): S37-S45, 2015.

20. Wang WM, Zhao ZL, Zhang WF, Zhao YF, Zhang L and Sun ZJ: Role of hypoxia-inducible factor-1 $\alpha$ and CD146 in epidermal growth factor receptor-mediated angiogenesis in salivary gland adenoid cystic carcinoma. Mol Med Rep 12: 3432-3438, 2015.

21. Kulsum U, Singh V, Sharma S, Srinivasan A, Singh TP and Kaur P: RASOnD-a comprehensive resource and search tool for RAS superfamily oncogenes from various species. BMC Genomics 12: 341, 2011.

22. Bouchard L, Thibault S, Guay SP, Santure M, Monpetit A, St-Pierre J, Perron P and Brisson D: Leptin gene epigenetic adaptation to impaired glucose metabolism during pregnancy. Diabetes Care 33: 2436-2441, 2010.

23. Huang da W, Sherman BT and Lempicki RA: Systematic and integrative analysis of large gene lists using DAVID bioinformatics resources. Nat Protoc 4: 44-57, 2009.

24. Baba SA, Mohiuddin T, Basu S, Swarnkar MK, Malik AH, Wani ZA, Abbas N, Singh AK and Ashraf N: Comprehensive transcriptome analysis of Crocus sativus for discovery and expression of genes involved in apocarotenoid biosynthesis. BMC Genomics 16: 698, 2015.

25. Munson ME: An improved technique for calculating relative response in cellular proliferation experiments. Cytometry A 77: 909-910, 2010.

26. Okada H, Ban S, Nagao S, Takahashi H, Suzuki H and Neilson EG: Progressive renal fibrosis in murine polycystic kidney disease: An immunohistochemical observation. Kidney Int 58: 587-597, 2000.

27. Zhao X, Harashima H, Dissmeyer N, Pusch S, Weimer AK, Bramsiepe J, Bouyer D, Rademacher S, Nowack MK, Novak B, et al: A general G1/S-phase cell-cycle control module in the flowering plant Arabidopsis thaliana. PLoS Genet 8: e1002847, 2012.

28. Chang CN, Feng MJ, Chen YL, Yuan RH and Jeng YM: $\mathrm{p} 15(\mathrm{PAF})$ is an $\mathrm{Rb} / \mathrm{E} 2 \mathrm{~F}$-regulated S-phase protein essential for DNA synthesis and cell cycle progression. PLoS One 8: e61196, 2013.

29. Paquin MC, Cagnol S, Carrier JC, Leblanc C and Rivard N: ERK-associated changes in E2F4 phosphorylation, localization and transcriptional activity during mitogenic stimulation in human intestinal epithelial crypt cells. BMC Cell Biol 14: 33, 2013.

30. Garneau H, Paquin MC, Carrier JC and Rivard N: E2F4 expression is required for cell cycle progression of normal intestinal crypt cells and colorectal cancer cells. J Cell Physiol 221: $350-358,2009$.

31. Oda A, Taniguchi $\mathrm{T}$ and Yokoyama M: Leptin stimulates rat aortic smooth muscle cell proliferation and migration. Kobe J Med Sci 47: 141-150, 2001.

32. Si HF, Li J, Lü XW and Jin Y: Suppressive effects of leflunomide on leptin-induced collagen I production involved in hepatic stellate cell proliferation. Exp Biol Med (Maywood) 232: 427-436, 2007.

33. Garofalo C, Koda M, Cascio S, Sulkowska M, Kanczuga-Koda L, Golaszewska J, Russo A, Sulkowski S and Surmacz E: Increased expression of leptin and the leptin receptor as a marker of breast cancer progression: Possible role of obesity-related stimuli. Clin Cancer Res 12: 1447-1453, 2006.

34. Simerly RB: Wired on hormones: Endocrine regulation of hypothalamic development. Curr Opin Neurobiol 15: 81-85, 2005.

35. Harrold JA: Leptin leads hypothalamic feeding circuits in a new direction. Bioessays 26: 1043-1045, 2004. 
36. Kitase Y, Yokozeki M,Fujihara S, Izawa T, Kuroda S, Tanimoto K, Moriyama K and Tanaka E: Analysis of gene expression profiles in human periodontal ligament cells under hypoxia: The protective effect of CC chemokine ligand 2 to oxygen shortage. Arch Oral Biol 54: 618-624, 2009.

37. Agostini $\mathrm{C}$ and Gurrieri C: Chemokine/cytokine cocktail in idiopathic pulmonary fibrosis. Proc Am Thorac Soc 3: 357-363, 2006.
38. Kim JW, Tchernyshyov I, Semenza GL and Dang CV: HIF-1-mediated expression of pyruvate dehydrogenase kinase: A metabolic switch required for cellular adaptation to hypoxia. Cell Metab 3: 177-185, 2006.

39. Xu J and Kisseleva T: Bone marrow-derived fibrocytes contribute to liver fibrosis. Exp Biol Med (Maywood) 240: 691-700, 2015. 